\title{
ORIGINAL
}

Igor I. Andrianov · Jan Awrejcewicz • Alexander A. Diskovsky

\section{Optimal design of a functionally graded corrugated cylindrical shell subjected to axisymmetric loading}

Received: 21 July 2017 / Accepted: 19 February 2018 / Published online: 27 February 2018

(C) The Author(s) 2018. This article is an open access publication

\begin{abstract}
In this work we consider an optimal design of corrugated cylindrical shells. The functionally graded (FG) corrugation with amplitude following a given shape is defined. The problem is solved as a linear one assuming the axially symmetric loading. The method of estimation of the stress-strain state and, consequently, the shell optimal design based on homogenization approach is proposed. The illustrative examples of the optimal FG corrugation shell subjected to hydrostatic load have demonstrated high efficiency of the employed method.
\end{abstract}

Keywords Cylindrical shell · Corrugation · Functionally graded structure · Homogenization approach · Optimal design

\section{Introduction}

Corrugation significantly changes the mechanical characteristics of shells, i.e., the bending stiffness can be increased in one direction, whereas the shell flexibility can be increased in other direction. In order to increase the load bearing capacity of longitudinally loaded cylindrical shells (columns, bodies of rockets), the corrugated process is usually employed in a circular direction. In the case of internal/external pressure, the longitudinally corrugated shell structures are applied. They are widely used in civil, naval, automotive, and aerospace engineering because of their superior properties. One can mention metal bellows working as elastic vessels that can be compressed when pressure is applied to the outside of the vessel, or extended under vacuum (Fig. 1). They are used, e.g., as piping expansion joint. A silo is a corrugated structure for storing bulk materials (Fig. 2). Silos are commonly used for bulk storage of grain, coal, cement, carbon black, woodchips, food products and sawdust. The mentioned shells are usually subjected to axially symmetric load. Though in the case of a bellow the essential role is played by nonlinearity of deformations, in the case of a silo the strain-stress problem can be considered in the framework of the linear theory.

I. I. Andrianov

Institut für Bauforschung, RWTH Aachen University, Schinkelstr. 3, 52062 Aachen, Germany

E-mail: andrianov@ibac.rwth-aachen.de

J. Awrejcewicz (ه)

Department of Automation, Biomechanics and Mechatronics, Lodz University of Technology, 1/15 Stefanowski St., 90-924 Lodz, Poland

E-mail: awrejcew@p.lodz.pl

\section{A. A. Diskovsky}

Department of Higher Mathematics, National Metallurgical Academy of Ukraine, 23 Gagarina Str., Dnepr 49005, Ukraine

E-mail: alex_diskovskiy@ukr.net 


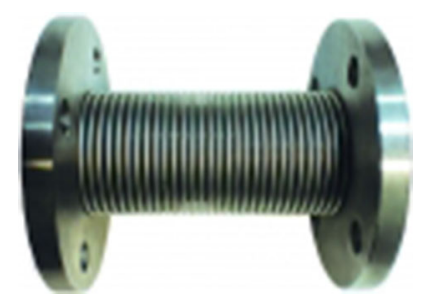

Fig. 1 Compensator of longitudinal extension of pipelines

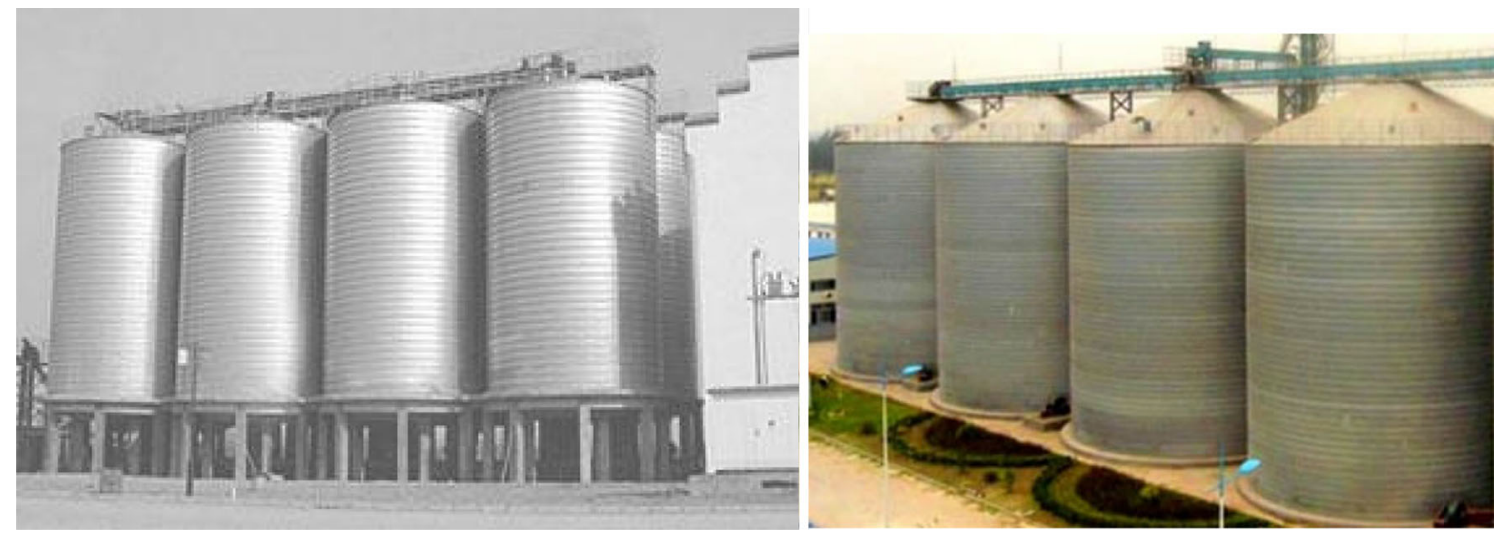

Fig. 2 Silos for storing fluid and loose materials

The methods of computation and optimization of corrugated shells have been considered mainly for the case of a regular corrugation. As an example, we can mention metallic corrugated core sandwich panels. In particular, an influence of different cores geometry has been taken into account, including square, triangular and trapezoidal cores [1,2]. An optimal shape of the corrugated profile under longitudinal deformation has been proposed in work [3], whereas the influence of the length and amplitude of corrugations has been studied in references $[4,5]$. On the other hand, it is possible to design more effective functionally graded (FG) corrugation, where both the amplitude and the step are changed by means of following a given rule [6,7]. The latter option of the optimal design has been investigated to a lesser extent. The reason is mainly motivated by occurrence of additional modeling and computational difficulties in comparison with the known problems exhibited by regularly graded corrugated structures. The usual equivalent plate model of corrugated structures [8-17] is not suitable for the FG corrugation. Although commercial codes allow one to analyze corrugated structures by approximation of corrugations using shell or solid elements [18-25], such approach is not practical due to a large number of corrugations.

Therefore, the key and most important problem is formulation and then effective solution of the problem of optimal design of the FG corrugated shells. Employment of numerical approaches for optimal design of FG corrugations implies occurrence of numerous difficulties in the sense of the time-consuming computations [26-33]. The homogenization approach seems to be promising in this case [34-37]. This asymptotic approach exploit the smallness of a single corrugation size with respect to the characteristic size of the corrugated structure. Namely, we use here one of the variants of the homogenization approach, modified to the needs of computation and optimal design of the FG structures [38-41].

Although the employed methodology of the paper is relatively simple, its technical realization is not easy. A corrugated shell is substituted by a certain orthotropic shell with the averaged characteristics. We use the homogenization approach, which is one of the most feasible methods for analysis of such transition. However, the application of the homogenization method to the corrugated shells requires much more attention and study than its application to, for instance ribbed or perforated shells or even composite materials. This problem does not belong to simple ones because the governing equations of the initial corrugated shell and its counterpart orthotropic model are derived with regard to different reference surfaces. In order to keep reliable transition into orthotropic shell, the initial governing relations should be projected onto the axes of the cylindrical surface. 


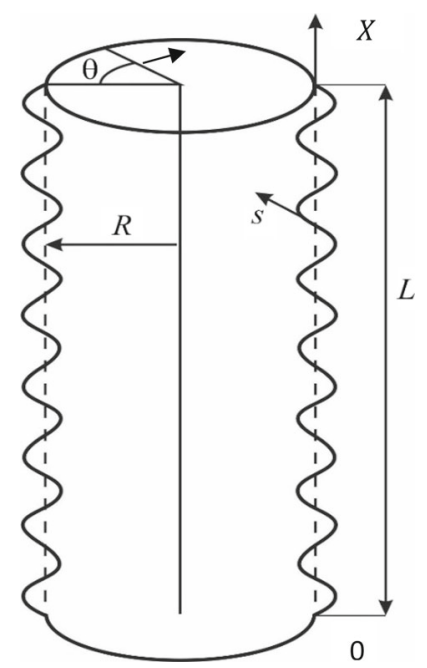

Fig. 3 Longitudinally corrugated cylindrical shell

In our case, it can be done only by splitting the initial/input relation into bending and tangential components which yields complicated analytical formulas. In the next step, the multiple homogenization approach is used to study the projectional equations. The problem of optimization is solved with the variational approach.

The presented case study validated our theoretical investigations.

The paper is organized as follows. First, we employ the fundamental relations with regard to axially symmetric deformation (Sect. 2). In Sect. 3, the homogenization of the fundamental relations of the axially symmetric deformations is carried out. Section 4 deals with an optimal design of a FG corrugation under action of the distributed loading. Section 5 presents an example of the optimization procedure when the shell is subjected to hydrostatic pressure. Finally, Sect. 6 presents concluding remarks.

\section{Basic relations for axially symmetric deformation}

We begin with a study of the longitudinally corrugated cylindrical shell shown in Fig. 3 .

In classical models of the shell, systems of coordinates coinciding with the lines of principal curvatures of shell middle surface $s, \theta$ are usually employed (Fig. 3). However, as it has been pointed out in references $[32,33]$, this system of coordinates is not optimal for the homogenization of the corrugated shells. Therefore, it is worth to derive the fundamental relations for the axially symmetric deformation of a longitudinally corrugated shell with respect to the non-dimensional coordinates of the surface $x=X / L, \theta$ depicted in Fig. 3 by dashed lines. Projections of displacements, internal stresses and moments onto the surface are treated as the unknown functions. Let us cut-out a fragment of the reference surface (Fig. 4) and let us derive the equilibrium equation of projection of this element onto the middle shell surface (Fig. 3) with respect to projection of internal forces and moments onto the axes $x, \theta$ :

$$
\begin{aligned}
& \frac{\mathrm{d} N_{x}}{\mathrm{~d} x}+q_{x} L=0 ; \quad \frac{\mathrm{d} Q_{x}}{\mathrm{~d} x}-N_{\theta}+q_{z} L=0 ; \\
& \frac{\mathrm{d} M_{x}}{\mathrm{~d} x}-Q_{x} L-\beta\left(M_{\theta}-z R N_{\theta}\right)+z q_{x} L^{2}=0,
\end{aligned}
$$

where $z=\frac{Z}{L} ; z=z(x)$ stands for a distance between points of the middle surface and the reference surface; $\beta=\frac{\mathrm{d} z}{\mathrm{~d} x} ; N_{x}, Q_{x}, N_{\theta}, M_{x}, M_{\theta}, q_{x}, q_{z}$ are the projections of internal forces, moments and external load on the axis of the reference surface (Figs. 5, 6):

$$
\begin{aligned}
N_{x} & =\left(N_{s}^{\prime} \cos \varphi+Q_{s}^{\prime} \sin \varphi\right) A_{2} ; \quad N_{\theta}=N_{\theta}^{\prime} A_{1} ; \\
Q_{x} & =\left(Q_{s}^{\prime} \cos \varphi-N_{s}^{\prime} \sin \varphi\right) A_{2} ; \quad Q_{\theta}=Q_{\theta}^{\prime} A_{1} \cos \varphi ; \\
M_{x} & =M_{s}^{\prime} A_{2}+z N_{x} ; \quad M_{\theta}=M_{\theta}^{\prime} A_{1}+z N_{\theta} ; \\
q_{z} & =\left(q_{\mathrm{n}} \cos \varphi-q_{\mathrm{t}} \sin \varphi\right) A_{2} ; \quad q_{x}=\left(q_{\mathrm{t}} \cos \varphi+q_{\mathrm{n}} \sin \varphi\right) A_{2} .
\end{aligned}
$$




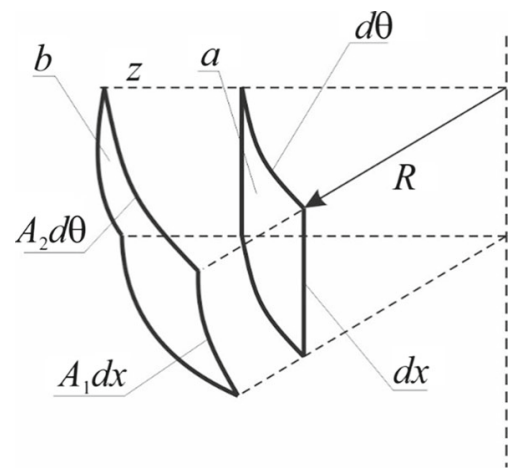

Fig. 4 Element of the reference surface $(a)$ and its projection onto the middle shell surface $(b)$

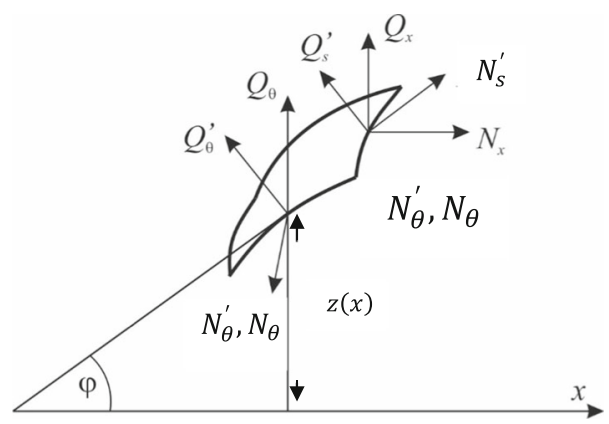

Fig. 5 Projection of internal forces onto the axis $s, \theta$ of the middle surface $\left(N_{s}^{\prime}, Q_{s}^{\prime}, N_{\theta}^{\prime}, Q_{\theta}^{\prime}\right)$ and onto the axis $x, \theta$ of the basic surface $\left(N_{x}, Q_{x}, N_{\theta}, Q_{\theta}\right) ; \varphi$ is the angle of inclination of a tangent to the middle surface; $z(x)$ is the distance to the basic surface

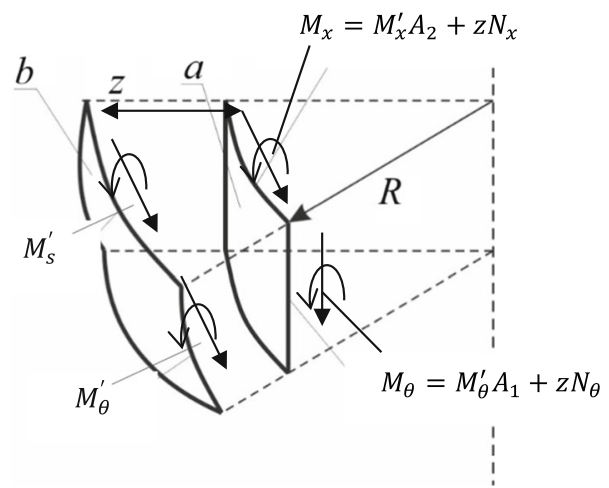

Fig. 6 Pictorial diagram of the moments action $\left(M_{s}^{\prime}, M_{\theta}^{\prime}\right.$ are the moments with respect to the axis of the middle surface $s, \theta$ and to the axis of the basic surface $x, \theta$, respectively)

Here, $N_{s}^{\prime}, Q_{s}^{\prime}, N_{\theta}^{\prime}, Q_{\theta}^{\prime}, M_{s}^{\prime}, M_{\theta}^{\prime}$ are the projections of internal forces and moments onto the axis of middle surface $s, \theta ; q_{\mathrm{n}}, q_{\mathrm{t}}$ stands for intensity of the normal and tangential middle surface load; $A_{1}=\sqrt{1+\beta^{2}}$; $A_{2}=1+z ; \sin \varphi=\frac{\beta}{A_{1}} ; \cos \varphi=\frac{1}{A_{1}} ; \varphi$ is the angle of inclination of a tangent to the middle surface (Fig. 5). form

The function $z=z(x)$ defines the geometry of corrugation profile and can be presented in the following

$$
z=H(x) \tilde{z}(n x),
$$

where $H(x)=\frac{h(x)}{L} ; h(x)$ is the amplitude of the corrugation profile; $\tilde{z}(n x)$ is the periodic function with period $n^{-1}$ and $n$ stands for a number of corrugation waves.

Let us consider projections of displacements of the points of the middle shell surface onto the axis of the reference surface $(u, v, w)$ shown in Fig. 7. Normal and tangential displacements with respect to the middle surface $\left(u^{\prime}, v^{\prime}, w^{\prime}\right)$ can be expressed via the introduced projections by the following formulas 


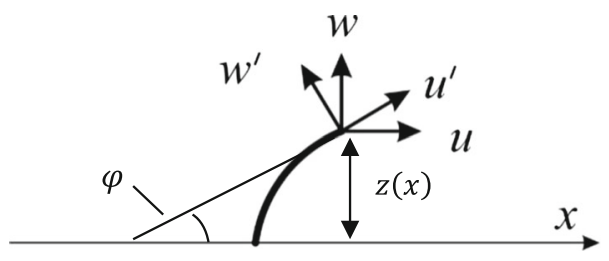

Fig. 7 Displacements being normal and tangential to the middle surface $\left(u^{\prime}, w^{\prime}\right)$ and their projections onto the axes of the reference surface $(u, w) ; \varphi$ is the angle of inclination of a tangent to the middle surface; $z(x)$ is the distance to the basic surface

$$
u^{\prime}=\frac{u+\beta w}{A_{1}} ; \quad v^{\prime}=v ; \quad w^{\prime}=\frac{w-\beta u}{A_{1}} .
$$

Substituting relations (6) into the known formulas of the linear axially symmetric deformation of a shell [42], the following formulas governing deformations and changes of the curvature of the middle surface via projections of displacements onto the axes of basic surface are obtained:

$$
\varepsilon_{s}=\frac{1}{A_{1}^{2} R}\left(\frac{\mathrm{d} u}{\mathrm{~d} x}+\beta \frac{\mathrm{d} w}{\mathrm{~d} x}\right) ; \quad \varepsilon_{\theta}=\frac{w}{R A_{2}} ; \quad \chi=\frac{1}{A_{1} R^{2}} \frac{\mathrm{d}}{\mathrm{d} x}\left(\frac{1}{A_{1}^{2}}\left(\frac{\mathrm{d} w}{\mathrm{~d} x}-\beta \frac{\mathrm{d} u}{\mathrm{~d} x}\right)\right) .
$$

Taking into account relations (2)-(7), physical relations for axially symmetric deformations can be recast to the following form

$$
\begin{aligned}
N_{x}-\beta Q_{x} & =\frac{E h}{1-v^{2}} A_{2}\left(\frac{1}{A_{1}^{2} R}\left(\frac{\mathrm{d} u}{\mathrm{~d} x}+\beta \frac{\mathrm{d} w}{\mathrm{~d} x}\right)+v \frac{w}{R A_{2}}\right) ; \\
M_{x}-z N_{x} & =\frac{D A_{2}}{R^{2} A_{1}} \frac{\mathrm{d}}{\mathrm{d} x}\left(\frac{1}{A_{1}^{2}}\left(\frac{\mathrm{d} w}{\mathrm{~d} x}-\beta \frac{\mathrm{d} u}{\mathrm{~d} x}\right)\right) ; \\
N_{\theta} & =\frac{E h}{1-v^{2}} \frac{1}{A_{2}}\left(A_{1} \frac{w}{R}+\frac{v}{A_{1}^{2} R}\left(\frac{\mathrm{d} u}{\mathrm{~d} x}+\beta \frac{\mathrm{d} w}{\mathrm{~d} x}\right)\right),
\end{aligned}
$$

where $D=\frac{E h^{3}}{12\left(1-v^{2}\right)} ; E, h, v$ are the Young's modulus, the shell thickness and the Poisson's ratio, respectively.

In what follows we split projections of displacements $u, w$ into two components: for the tangential deformations $\left(u^{t}, w^{t}\right)$ and for the bending ones $\left(u^{b}, w^{b}\right)$

$$
u=u^{t}+u^{b} ; \quad w=w^{t}+w^{b},
$$

where

$$
\begin{aligned}
& \frac{\mathrm{d} u^{b}}{\mathrm{~d} x}+\beta \frac{\mathrm{d} w^{b}}{\mathrm{~d} x}=0 \\
& \frac{\mathrm{d}}{\mathrm{d} x}\left(\frac{1}{A_{1}^{2}}\left(\frac{\mathrm{d} w^{t}}{\mathrm{~d} x}-\beta \frac{\mathrm{d} u^{t}}{\mathrm{~d} x}\right)\right)=0
\end{aligned}
$$

\section{Homogenization of relations of axially symmetric deformations}

The so far obtained relations present a PDE with rapidly oscillating coefficients. This kind of problem can be efficiently solved by the homogenization method based on the two-scale asymptotic expansions [34-37]. Let us introduce a variable $\xi=n x$, which is assumed to be independent on $x$, hence

$$
\frac{\mathrm{d}}{\mathrm{d} x}=\frac{\partial}{\partial x}+n \frac{\partial}{\partial \xi}
$$


Projections of forces and moments are expanded into the following series

$$
N_{x}=\sum_{0}^{\infty} n^{-k} N_{x k}(x, \xi) ; \quad M_{x}=\sum_{0}^{\infty} n^{-k} M_{x k}(x, \xi) ; \quad Q_{x}=\sum_{0}^{\infty} n^{-k} Q_{x k}(x, \xi),
$$

where $N_{x k} ; Q_{x k} ; M_{x k}$ stand for periodic functions with respect to $\xi$ and have the period 1.

Substituting (14) and (15) into equilibrium equations and splitting with respect to the parameter $n^{-1}$, one gets

$$
\begin{aligned}
& \frac{\partial N_{x 0}}{\partial \xi}=\frac{\partial Q_{x 0}}{\partial \xi}=0 ; \quad \frac{\partial M_{x 0}}{\partial \xi}=0 ; \\
& \frac{\partial N_{x 1}}{\partial \xi}+\frac{\partial N_{x 0}}{\partial x}+q_{x} L=0 ; \\
& \frac{\partial Q_{x 1}}{\partial \xi}+\frac{\partial Q_{x 0}}{\partial x}-N_{\varphi 0}+q_{z} L=0 ; \\
& \frac{\partial M_{x 1}}{\partial \xi}+\frac{\partial M_{x 0}}{\partial x}-Q_{x 0} L-\beta\left(M_{\varphi 0}-z N_{\varphi 0}\right)=0 ; \\
& \frac{\partial N_{x 2}}{\partial \xi}+\frac{\partial N_{x 1}}{\partial x}=0 .
\end{aligned}
$$

Integrating Eqs. (17)-(20) in $\xi$ from 0 to 1 and taking into account periodicity of the functions $N_{x 1}$, $Q_{x 1}, M_{x 1}$, the following homogenized equations are obtained

$$
\begin{aligned}
& \frac{\mathrm{d} N_{x 0}}{\mathrm{~d} x}+\operatorname{Lm}\left(q_{x}\right)=0, \\
& \frac{\mathrm{d}^{2} M_{x 0}}{\mathrm{~d} x^{2}}-R m\left(N_{\varphi 0}\right)-\frac{\mathrm{d}}{\mathrm{d} x} m\left(\beta\left(M_{\varphi 0}-z N_{\varphi 0}\right)\right)+L^{2} m\left(q_{z}\right)=0, \\
& \frac{\mathrm{d}}{\mathrm{d} x} m\left(N_{x 1}\right)=0,
\end{aligned}
$$

where $m(\cdots)=\int_{0}^{1}(\cdots) \mathrm{d} \xi$

Components of the series for $N_{x}$ (15) can be obtained from Eqs. (17), (21) by employing simple integration:

$$
\begin{aligned}
& N_{x 0}=N_{x}^{c}-L \int m\left(q_{x}\right) \mathrm{d} x ; \\
& N_{x 1}=L \int\left(m\left(q_{x}\right)-q_{x}\right) \mathrm{d} \xi+N_{x 10}(x),
\end{aligned}
$$

where $N_{x}^{c}$ is the force on the boundary $(x=0)$, and the integration constant $N_{x 10}(x)$ is yielded by the Eq. (23), i.e., we have

$$
N_{x 10}=C-\operatorname{Lm}\left(\int\left(m\left(q_{x}\right)-q_{x}\right) \mathrm{d} \xi\right) .
$$

An arbitrary constant $C$ is defined by the boundary conditions.

Since the longitudinal deformation implied by the external load $q_{x}$ is statically determinate, we study only the case of bending deformation governed by the load $q_{z}$. For this purpose, the bending components of projection of the displacements $u^{b}, w^{b}$ are presented as follows [30]

$$
\begin{aligned}
u^{b} & =n^{-1} u_{0}^{b}(\xi, x)+n^{-2} u_{1}^{b}(\xi, x)+\cdots ; \\
w^{b} & =w_{0}^{b}(\xi, x)+n^{-2} w_{1}^{b}(\xi, x)+\cdots,
\end{aligned}
$$

where $u_{k}^{b}, w_{k}^{b}$ are periodic functions in $\xi$ with period 1 . 
Substituting the series (15) and (27) into physical relations (9), (10) and taking into account conditions (12), (14), the following equations are derived:

$$
\begin{aligned}
w_{0}^{b} & =w_{0}^{b}(x) ; \quad u_{0}^{b}=-n z \frac{\mathrm{d} w_{0}^{b}}{\mathrm{~d} x} ; \\
M_{x 0} & =D \frac{A_{2}}{R^{2} A_{1}}\left(\frac{\mathrm{d}^{2} w_{0}^{b}}{\mathrm{~d} x^{2}}+\frac{\partial^{2} w_{1}^{b}}{\partial \xi^{2}}\right) ; \\
N_{\varphi 0} & =E h A_{1} \frac{w_{0}^{b}}{R A_{2}} .
\end{aligned}
$$

Equilibrium equations (16), (22), taking into account (28), are recast to the following forms

$$
\begin{aligned}
& \frac{\partial}{\partial \xi}\left(\frac{A_{2}}{A_{1}} \frac{\partial^{2} w_{1}^{b}}{\partial \xi^{2}}\right)+\frac{\partial}{\partial \xi}\left(\frac{A_{2}}{A_{1}}\right) \frac{\mathrm{d}^{2} w_{0}^{b}}{\mathrm{~d} x^{2}}=0 \\
& \frac{\partial^{2}}{\partial x^{2}}\left(\frac{A_{2}}{A_{1}}\left(\frac{\mathrm{d}^{2} w_{0}^{b}}{\mathrm{~d} x^{2}}+\frac{\partial^{2} w_{1}^{b}}{\partial \xi^{2}}\right)\right)+m\left(\frac{A_{1}}{A_{2}}\right) \frac{E h}{D} w_{0}^{b}=\frac{L^{2} m\left(q_{z}\right)}{D} .
\end{aligned}
$$

Homogenization of the Eq. (28) and the periodicity condition of the function $w_{1}^{b}$ yield

$$
\frac{A_{2}}{A_{1}}\left(\frac{\mathrm{d}^{2} w_{0}^{b}}{\mathrm{~d} x^{2}}+\frac{\partial^{2} w_{1}^{b}}{\partial \xi^{2}}\right)=\left(m\left(\frac{A_{1}}{A_{2}}\right)\right)^{-1} \frac{\mathrm{d}^{2} w_{0}^{b}}{\mathrm{~d} x^{2}} .
$$

Employing relation (31), the equilibrium equations (30) can be transformed to the following form

$$
\frac{\mathrm{d}^{2}}{\mathrm{~d} x^{2}}\left(\frac{1}{m\left(\frac{A_{1}}{A_{2}}\right)} \frac{\mathrm{d}^{2} w_{0}^{b}}{\mathrm{~d} x^{2}}\right)+m\left(\frac{A_{1}}{A_{2}}\right) \frac{E h}{D} w_{0}^{b}=\frac{L^{2} m\left(q_{z}\right)}{D} .
$$

Equation (32) describes the axially symmetric deformation of an orthotropic cylindrical shell of the radius $R$, the elastic characteristics of which present the averaged characteristics of the studied corrugated cylindrical shell. If we pass to a regular corrugation with the constant amplitude and take $A_{2}=1$ in Eq. (32), it coincides with the obtained in $[5,6]$. In practice, since for the majority of the corrugated cylinders the following inequality holds: $\max z \ll 1$; hence, we further assume $A_{2}=1$. One of the important advantages of our approach in comparison with the structurally orthotropic theory [3] consists in the possibility of improvement of the obtained averaged solution. For instance, relation (31) allows one to express $w_{1}^{b}$ by $w_{0}^{b}$

$$
\frac{\partial^{2} w_{1}^{b}}{\partial \xi^{2}}=\frac{A_{1}-m\left(A_{1}\right)}{m\left(A_{1}\right)} \frac{\mathrm{d}^{2} w_{0}^{b}}{\mathrm{~d} x^{2}} .
$$

Furthermore, one can employ periodicity of the functions $w_{1}^{b}$ with respect to $\xi$ while integrating the Eq. (33). The fundamental benefit of the proposed method is the possibility of obtaining the effective solution to the problem of optimal design of a FG corrugated shells, since the control function $H(x)$ is contained explicitly in the coefficients of the equilibrium equations (32).

\section{Optimal design of the FG corrugated shell under distributed loading}

One of the key design specifications of the considered corrugated structures (Fig. 2) is their stiffness. It is important to ensure maximum rigidity of the designed structures with minimal weight. Weight of corrugated cylinders (Fig. 3), for a given thickness of the shell wall, is determined by the length of the generatrix playing the role of a constraint. In a number of works (see [42] and the references cited therein), external loading has been employed as a measure of stiffness properties of structures. One may proceed in a way similar to the mentioned one, i.e., to minimize weight for a given stiffness of a studied structure. 
Obviously, for the optimal FG corrugation, the change in the corrugation parameters must correspond to changes in the intensity of the external load. Since the distributed load intensity (in the majority of practical cases) varies smoothly, then we can consider the case of slowly changing corrugation amplitude

$$
\frac{\mathrm{d} H}{\mathrm{~d} x} \sim H
$$

Taking into account (14), (34), the following approximation holds

$$
A_{1} \approx \sqrt{1+\left(n H \frac{\mathrm{d} \tilde{z}}{\mathrm{~d} \xi}\right)^{2}}
$$

Let us consider a design of an optimal profile of the variable amplitude $H(x)$, which guarantees the largest stiffness of the corrugated cylinder with the clamped $(x=0)$ and free $(x=1)$ edges subjected to axially symmetric normal load $q(x)$. Observe that the choice of the boundary conditions does not influence our investigations, i.e., other boundary conditions can be taken.

Since the geometric conditions yield $m\left(A_{1}\right) \geq 1$, the auxiliary control function $\alpha(x)$, is introduced by means of the following relation

$$
m\left(A_{1}\right)=1+\alpha^{2},
$$

whereas the value $\alpha=0$ corresponds to the smooth shell.

We take the flexibility governed by (36) as the stiffness condition, whereas the length of the curvilinear axis of the corrugation profile (38) plays the role of a constraint. As a result, our problem is recast to the following one

$$
\begin{aligned}
& I=\int_{0}^{1} \bar{q} w \mathrm{~d} x \rightarrow \min _{\beta} ; \\
& I_{1}=\int_{0}^{1}\left(1+\alpha^{2}\right) \mathrm{d} x=S \\
& \frac{\mathrm{d}^{2}}{\mathrm{~d} x^{2}}\left(\frac{1}{1+\alpha^{2}} \frac{\mathrm{d}^{2} w}{\mathrm{~d} x^{2}}\right)+\left(1+\alpha^{2}\right) \rho w=\bar{q} ; \\
& \text { for }=0: w=0, \quad \frac{\mathrm{d} w}{\mathrm{~d} x}=0 ; \quad \text { for } x=1: \frac{\mathrm{d}^{2} w}{\mathrm{~d} x^{2}}=0 ; \quad \frac{\mathrm{d}^{3} w}{\mathrm{~d} x^{3}}=0
\end{aligned}
$$

where $w=w_{0}^{b} ; \rho=\frac{12\left(1-v^{2}\right)}{h^{2}} ; \bar{q}=\frac{L^{2} m\left(q_{z}\right)}{D}$.

Let us write expressions for the first variations of the integrals (37), (38), and then the variational form of Eq. (39) and boundary conditions (40) as follows

$$
\begin{aligned}
& \delta I=\int_{0}^{1} \bar{q} \delta w \mathrm{~d} x ; \quad \delta I_{1}=\int_{0}^{1} 2 \alpha \delta \alpha \mathrm{d} x \\
& \frac{\mathrm{d}^{2}}{\mathrm{~d} x^{2}}\left(\frac{1}{1+\alpha^{2}} \frac{\mathrm{d}^{2} \delta w}{\mathrm{~d} x^{2}}\right)-\frac{\mathrm{d}^{2}}{\mathrm{~d} x^{2}}\left(\frac{2 \alpha \delta \alpha}{\left(1+\alpha^{2}\right)^{2}} \frac{\mathrm{d}^{2} w}{\mathrm{~d} x^{2}}\right)+\left(1+\alpha^{2}\right) \rho \delta w+2 \alpha \delta \alpha \rho w=0 .
\end{aligned}
$$

Multiplying the left-hand side of Eq. (42) by the conjugate variable $\omega$, we carry out integration from 0 to 1. In the next step, we integrate the first term by parts four times, and the second term two times. The outer integral terms will be equal to zero if for the conjugate variable and for the control function the following boundary conditions will be employed

$$
x=0: \omega=0, \quad \frac{\mathrm{d} \omega}{\mathrm{d} x}=0 ; \quad \text { for } x=1: \frac{\mathrm{d}^{2} \omega}{\mathrm{d} x^{2}}=0 ; \quad \frac{\mathrm{d}^{3} \omega}{\mathrm{d} x^{3}}=0 .
$$

As a result, we obtain

$$
\int_{0}^{1}\left(\left(\frac{\mathrm{d}^{2}}{\mathrm{~d} x^{2}}\left(\frac{1}{1+\alpha^{2}} \frac{\mathrm{d}^{2} \omega}{\mathrm{d} x^{2}}\right)+\left(1+\alpha^{2}\right) \rho \omega\right) \delta w-2 \alpha\left(\frac{1}{\left(1+\alpha^{2}\right)^{2}} \frac{\mathrm{d}^{2} w}{\mathrm{~d} x^{2}} \frac{\mathrm{d}^{2} \omega}{\mathrm{d} x^{2}}-\omega \rho w\right) \delta \alpha\right) \mathrm{d} x=0
$$


In order to finding a local minimum (37) of the function $\alpha(x)$ under constraint (38), the method of Lagrange multipliers is introduced:

$$
\int_{0}^{1}\left(\left(\frac{\mathrm{d}^{2}}{\mathrm{~d} x^{2}}\left(\frac{1}{1+\alpha^{2}} \frac{\mathrm{d}^{2} \omega}{\mathrm{d} x^{2}}\right)+\left(1+\alpha^{2}\right) \rho \omega+\bar{q}\right) \delta w-2 \alpha\left(\frac{1}{\left(1+\alpha^{2}\right)^{2}} \frac{\mathrm{d}^{2} w}{\mathrm{~d} x^{2}} \frac{\mathrm{d}^{2} \omega}{\mathrm{d} x^{2}}-\omega \rho w-\lambda\right) \delta \alpha\right) \mathrm{d} x=0,
$$

where $\lambda$ stands for the Lagrange multiplier.

Formula (45) yields the equation governing behavior of the conjugate variable

$$
\frac{\mathrm{d}^{2}}{\mathrm{~d} x^{2}}\left(\frac{1}{1+\alpha^{2}} \frac{\mathrm{d}^{2} \omega}{\mathrm{d} x^{2}}\right)+\left(1+\alpha^{2}\right) \rho \omega+\bar{q}=0
$$

The necessary condition of optimality is

$$
2 \alpha\left(\frac{1}{\left(1+\alpha^{2}\right)^{2}} \frac{\mathrm{d}^{2} w}{\mathrm{~d} x^{2}} \frac{\mathrm{d}^{2} \omega}{\mathrm{d} x^{2}}-\omega \rho w-\lambda\right)=0 .
$$

Comparing the boundary value problems (39), (40) and (43), (46), we get

$$
\omega=-w
$$

The necessary condition of optimality (48), taking into account (47), can be transformed to the following form

$$
2 \alpha\left(\frac{1}{\left(1+\alpha^{2}\right)^{2}}\left(\frac{\mathrm{d}^{2} w}{\mathrm{~d} x^{2}}\right)^{2}-\rho w^{2}+\lambda\right)=0
$$

If the optimality condition (49) is satisfied only due to the second factor, then

$$
\frac{1}{\left(1+\alpha^{2}\right)^{2}}\left(\frac{\mathrm{d}^{2} w}{\mathrm{~d} x^{2}}\right)^{2}-\rho w^{2}+\lambda=0
$$

However, in this case, the boundary conditions (40) are not satisfied. This motivates us to take the target function $\alpha(x)$ as the piecewise continuous, $\alpha=0$ at $x \in\left(0, x_{1}\right) \cup\left(x_{2}, 1\right)$. Then, equilibrium equations (39) at $x \in\left(0, x_{1}\right) \cup\left(x_{2}, 1\right)$ can be written as follows

$$
\frac{\mathrm{d}^{4} w_{b}^{(i)}}{\mathrm{d} x^{4}}+\frac{E h}{D} w_{b}^{(i)}=\frac{L^{2}\left(q_{z}\right)}{D}, \quad i=1,2,
$$

where $w_{b}^{(1)}, w_{b}^{(2)}$ are the deflection regarding the intervals $\left(0, x_{1}\right)$ and $\left(x_{2}, 1\right)$.

We take the conditions of deflection and deformation continuity in the points $x_{1}, x_{2}$ (the WeierstrassErdmann conditions [42]) as boundary conditions for obtained system (39), (50), (51) and obtain

$$
\begin{aligned}
& \lim _{x \rightarrow x_{1,2}-0} w=\lim _{x \rightarrow x_{1,2}+0} w ; \quad \lim _{x \rightarrow x_{1,2}-0} \frac{\mathrm{d} w}{\mathrm{~d} x}=\lim _{x \rightarrow x_{1,2}+0} \frac{\mathrm{d} w}{\mathrm{~d} x} \\
& \lim _{x \rightarrow x_{1,2}-0} \frac{\mathrm{d}^{2} w}{\mathrm{~d} x^{2}}=\lim _{x \rightarrow x_{1,2}+0} \frac{\mathrm{d}^{2} w}{\mathrm{~d} x^{2}} .
\end{aligned}
$$

The Lagrange multiplier $\lambda$ is defined through the isoperimetric condition (38), which owing to $\alpha=0$ at $x \in$ $\left(0, x_{1}\right) \cup\left(x_{2}, 1\right)$, takes the following form

$$
\int_{x_{1}}^{x_{2}}\left(1+\alpha^{2}\right) \mathrm{d} x=S+x_{2}-x_{1}-1
$$

In what follows we investigate physical meaning of the optimality condition (49). We obtain the deflection of the FG part of the corrugated cylinder in the interval $\left(x_{1}, x_{2}\right)$ from the optimality condition (49). It should be emphasized that the characteristic property of optimal FG structures does not depend on the shape of continuously distributed external load (it does not appear in the optimality conditions). The magnitude of external 
load influences only the magnitude of deformation. As an expression satisfying the optimality condition, we choose the particular solution of the equilibrium equation (39) $w=$ const, then from (49) we obtain

$$
w= \pm \sqrt{\frac{\lambda}{\rho}} .
$$

The constancy of the deformation of the FG part is an interesting feature of optimal designs of FG structures. Substituting expression (54) into Eq. (39), the following auxiliary target function is obtained

$$
\alpha^{2}= \pm \frac{\bar{q}}{\bar{\lambda}}-1
$$

where $\bar{\lambda}=\sqrt{\rho \lambda}$; the sign is chosen depending on the direction of the external load $q$.

After obtaining the auxiliary target function $\alpha^{2}(x)$, the optimal profile $H(x)$ defined on the intervals $\left(x_{1}, x_{2}\right)$ can be yielded by the relation (35), (36)

$$
\int_{0}^{1} \sqrt{1+\left(n H \frac{\mathrm{d} \tilde{z}}{\mathrm{~d} \xi}\right)^{2}} \mathrm{~d} \xi= \pm \frac{\bar{q}}{\bar{\lambda}}
$$

In particular, in the case of sinusoidal shape of the profile,

$$
\tilde{z}=\sin (2 \pi n x)
$$

Eq. (56) takes the following form

$$
E\left(\sqrt{-k^{2}}\right)= \pm \frac{\pi \bar{q}}{2 \bar{\lambda}}
$$

where $\boldsymbol{k}=\boldsymbol{n} \boldsymbol{H}$ and $\boldsymbol{E}($.) is the complete elliptic integral of the second kind.

For corrugated containers (Fig. 2) as a rule $k<1$, i.e., corrugation amplitude is less than its step. Then, in order to solve Eq. (58), one may use the decomposition of elliptic integrals in powers of $k$ as follows

$$
1+\frac{1}{4} k^{2}-\frac{3}{64} k^{4}+0\left(k^{6}\right)= \pm \frac{\pi \bar{q}}{2 \bar{\lambda}}
$$

Taking into account only to first two terms of the series (59), we get

$$
H(x)= \pm \frac{2}{n} \sqrt{ \pm \frac{\pi \bar{q}(x)}{2 \bar{\lambda}}-1}
$$

The sign under the root is chosen in a way depending on the external load $q$ direction to satisfy a positive value under the root term. The sign in front of the root defines two possible optimal designs differing in the direction of convexity of the corrugation first half-wave. Therefore, in the case of the sinusoidal shape of corrugation, the variable amplitude of its FG profile is governed by the following equation

$$
H(x)=\left\{\begin{array}{c}
0, x \in\left(0, x_{1}\right) \cup\left(x_{2}, l\right) \\
\pm \frac{2}{n} \sqrt{ \pm \frac{\pi \bar{q}}{2 \bar{\lambda}}-1}, x \in\left(x_{1}, x_{2}\right)
\end{array}\right.
$$




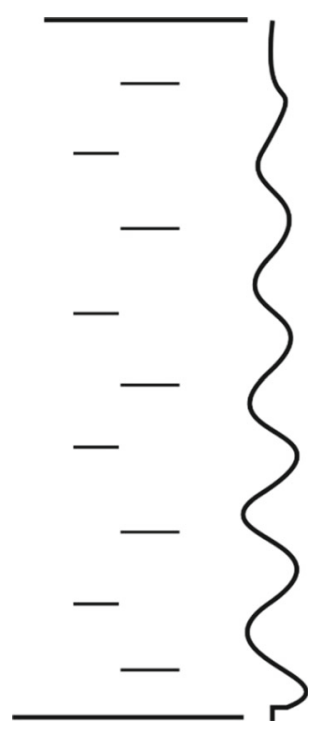

Fig. 8 Optimal profile of the sinusoidal corrugation $z=H(x) \sin 2 \pi n x$ under the hydrostatic loading for $\mathrm{n}=6 ; S=3 \pi$ (corrugation amplitude $H(x)$ varies according to the formula (60), whereas the upper and lower edges of the plots have zero amplitude)

\section{Example of optimization}

We consider the case of the hydrostatic load $\bar{q}=\gamma(1-x), \gamma=$ const. If we take the FG sinusoidal profile (57), then the optimal rule of variation of the amplitude has the form (61), and its design requires definition of the parameters $x_{1}, x_{2}, \bar{\lambda}$ for the given shell characteristics $n, S$. In order to define sizes of the smooth (not corrugated) border parts of the shell $\left(0, x_{1}\right),\left(1-x_{2}, 1\right)$, the deflections $w_{b}^{(i)}$, being solutions to Eq. (51), are assumed in the following way

$$
w_{i}=\sum_{j=1}^{4} C_{j i} K_{j}(\eta x)+\frac{\bar{q}}{\rho},
$$

where: $i=1,2 ; C_{j i}$ are the integration constants; $\eta=\sqrt[4]{\rho / 4} ; K_{j}(\eta x)$ are the Krylov functions [43] of the following form

$$
\begin{aligned}
& K_{1}(x)=\cos h(x) \cos (x) ; \quad K_{2}(x)=0.5(\cos h(x) \sin (x)+\sin h(x) \cos (x)) ; \\
& K_{3}(x)=0.5 \sin h(x) \sin (x) ; \quad K_{4}(x)=0.25(\cos h(x) \sin (x)-\sin h(x) \cos (x)) .
\end{aligned}
$$

Substituting formulas (54), (62) into the continuity conditions (52), boundary conditions (40), and the isoperimetric conditions (53), we obtain a system of equations to find $C_{j, i}, x_{1}, x_{2}, \bar{\lambda}$. Solving the obtained system the following conclusion can be yielded: the length of the upper smooth part of the free edge $1-x_{2} \leq$ $x \leq 1$ is practically equal to zero, whereas the length of the below smooth part $x_{1}$ decreases while increasing $S$. The optimal profile of the sinusoidal corrugation (57), (60) for $n=6 ; S=3 \pi$ is shown in Fig. 8.

The maximum deflection (54) of the optimally FG corrugated sheath (Fig. 8) was compared with the maximum deflection of a regularly corrugated shell having the same length of the curvilinear axis. The maximum deflection of the regular shell is defined with the help of the homogenized equation (39) keeping constant corrugation amplitude of $H=0.0436$. The carried-out analysis has shown that the relative decrease in the maximum deflection achieves about $50 \%$.

In the case of the optimal profile (Fig. 8), we take minus sign in front of the root in Eq. (60). If we take the flexibility (37) as the optimality criterion and we do not take into account the derivatives (stresses), then the sign choice does not play any role. If in the constraints or the optimality criteria the derivatives participate, then the optimality problem must be solved with allowance for corrections to the averaged solution. In the latter case, the sign in relations (60) plays an important role, since it defines the direction of convexity of the first corrugation half-wave, which consequently influences the local distribution of stresses. 


\section{Concluding remarks}

Overall, the carried-out optimization of the FG corrugation with variable amplitude has been realized by using two approaches: isolating the smooth sections near the edges of the shell and changing the height of the corrugation according to the law determined by the change in the load.

The first approach only works for uniform loads. Using this approach could, for example, increase the sensitivity of the FG round corrugated diaphragms [5]. The second approach is aimed at changing the height of the corrugation, and it is especially effective for non-uniform external load like hydrostatic loading, wind, etc. It can be expected that the use of the FG corrugation with variable amplitude would be effective also in the problems of stability of the corrugated structures. However, it should be mentioned that the employment of FG corrugation increases the cost of producing such structures.

It should be also emphasized that optimal FG corrugation, for any type of smoothly distributed external load and any boundary conditions, the deflection FG corrugated part will be uniform (55). The influence of the type of loading and the boundary conditions is expressed in the law of variation of the corrugation amplitude (60), in the sizes of the smooth border parts of the shell, and in the uniform deflection of the corrugated part.

Open Access This article is distributed under the terms of the Creative Commons Attribution 4.0 International License (http:// creativecommons.org/licenses/by/4.0/), which permits unrestricted use, distribution, and reproduction in any medium, provided you give appropriate credit to the original author(s) and the source, provide a link to the Creative Commons license, and indicate if changes were made.

\section{References}

1. Tian, Y.S., Lu, T.J.: Optimal design of compression corrugated panels. Thin Walled Struct. 43, 477-498 (2005)

2. Liang, C.-C., Yang, M.-F., Wu, P.-W.: Optimum design of metallic corrugated core sandwich panels subjected to blast loads. Ocean Eng. 28(7), 825-861 (2001)

3. Syerko, E., Diskovsky, A.A., Andrianov, I.V., Comas-Cardona, S., Binetruy, C.: Corrugated beams mechanical behavior modeling by the homogenization method. Int. J. Solids Struct. 50, 928-936 (2013)

4. Daxner, T., Flatscher, T., Rammerstorfer, F.G.: Optimum design of corrugated board under buckling constraints. In: 7th World Congress on Structural and Multidisciplinary Optimization. COEX Seoul, 21 May-25 May 2007, Korea, pp. 349-358 (2007)

5. Andrianov, I.V., Diskovsky, A.A., Syerko, E.: Optimal design of a circular diaphragm using the homogenization approach. Math. Mech. Solids 22(3), 283-303 (2017)

6. Andrianov, I.V., Awrejcewicz, J., Diskovsky, A.A.: Optimal design of a functionally graded corrugated rod subjected to longitudinal deformation. Arch. Appl. Mech. 85, 303-314 (2015)

7. Andreeva, L.E.: Elastic elements of instruments. Israel program for scientific translations (1966)

8. Arkhangelskii, A.F., Gorbachev, V.I.: Effective characteristics of corrugated plates. Mech. Solids 42, 447-462 (2007)

9. Bartolozzi, G., Pierini, M., Orrenius, U., Baldanzini, N.: An equivalent material formulation for sinusoidal corrugated cores of structural sandwich panels. Compos. Struct. 100, 173-185 (2013)

10. Ye, Z., Berdichevsky, V.L., Yu, W.: An equivalent classical plate model of corrugated structures. Int. J. Solids Struct. 51(11), 2073-2083 (2014)

11. Briassoulis, D.: Equivalent orthotropic properties of corrugated sheets. Compos. Struct. 23, 129-138 (1986)

12. Carlsson, L.A., Nordstrand, T., Westerlind, B.: On the elastic stiffnesses of corrugated core sandwich. J. Sandw. Struct. Mater. 3, 253-267 (2001)

13. Davalos, J.F., Qiao, P.Z., Xu, X.F., Robinson, J., Barth, K.E.: Modeling and characterization of fiber-reinforced plastic honeycomb sandwich panels for highway bridge applications. Compos. Struct. 52, 441-452 (2001)

14. Lau, J.H.: Stiffness of corrugated plate. J. Eng. Mech. Div. 107, 271-275 (1981)

15. Luo, S., Suhling, J.C.: The bending stiffnesses of corrugated board. Mech. Cell Mater. 145, 15-26 (1992)

16. Xia, Y., Friswell, M.I., Saavedra Flores, E.I.: Equivalent models of corrugated panels. Int. J. Solids Struct. 49(13), 1453-1462 (2012)

17. Liew, K.M., Peng, L.X., Kitipornchai, S.: Nonlinear analysis of corrugated plates using a FSDT and a mesh free method. Comput. Methods Appl. Mech. Eng. 196, 2358-2376 (2007)

18. Wennberg, D., Wennhage, P., Stichel, S.: Orthotropic models of corrugated sheets in finite element analysis. ISRN Mech. Eng. 2011, 9 (2011)

19. Talbi, N., Batti, A., Ayad, R., Guo, Y.Q.: An analytical homogenization model for finite element modelling of corrugated cardboard. Compos. Struct. 88, 280-289 (2009)

20. Aberg, M., Gudmundson, P.: The usage of standard finite element codes for computation of dispersion relations in materials with periodic microstructure. J. Acoust. Soc. Am. 102(4), 2007-2013 (1997)

21. Samanta, A., Mukhopadhyay, M.: Finite element static and dynamic analyses of folded plates. Eng. Struct. 21, 277-287 (1999)

22. Haj-Ali, R., Choi, J., Wei, B.S., Popil, R., Schaepe, M.: Refined nonlinear finite element models for corrugated fiberboards. Compos. Struct. 87, 321-333 (2009)

23. Gentilinia, C., Nobilea, L., Seffen, K.A.: Numerical analysis of morphing corrugated plates. Procedia Eng. 1, 79-82 (2009) 
24. Davalos, J.F., Qiao, P.Z., Xu, X.F., Robinson, J., Barth, K.E.: Modeling and characterization of fiber-reinforced plastic honeycomb sandwich panels for highway bridge applications. Compos. Struct. 52, 441-452 (2001)

25. Bisagni, C., Vescovini, R.: Fast tool for buckling analysis and optimization of stiffened panels. J. Aircr. 46(6), 2041-2053 (2009)

26. Lamberti, L., Venkataraman, S., Haftka, R.T., Johnson, T.F.: Preliminary design optimization of stiffened panels using approximate analysis models. Int. J. Numer. Methods Eng. 57(10), 1351-1380 (2003)

27. Liang, C.C., Yang, M.F., Wu, P.W.: Optimum design of metallic corrugated core sandwich panels subjected to blast loads. Ocean Eng. 28, 825-861 (2001)

28. Johnson, Jr. R.: Design and fabrication of a ring-stiffened graphite-epoxy corrugated cylindrical shell. NASA CR-3026 (1978)

29. Erdal, F., Dogan, E., Tunca, O., Tas, S.: Optimum design of corrugated web beams using stochastic search techniques. Int. J. Comput. Civ. Struct. Eng. 3(1), 104-108 (2016)

30. Alshabatat, N.: Design of corrugated plates for optimal fundamental frequency. Adv. Acoust. Vib. 2016, 9 (2016)

31. Dayyani, I., Woods, B.K.S., Friswell, M.I., Khodaparast, H.H.: The optimal design of a coated corrugated skin for the fish BAC morphing wing. In: ICAST 2013: 24th International Conference on Adaptive Structures and Technologies, October 7-9, Aruba, pp. 1-11 (2013)

32. Andrianov, I.V., Diskovsky, A.A., Kholod, E.G.: Homogenization method in the theory of corrugated plates. Tech. Mech. 18, 123-133 (1998)

33. Andrianov, I.V., Awrejcewicz, J., Diskovsky, A.A.: Asymptotic investigation of corrugated elements with quasi-periodic structures. In: Proceedings of 10th Conference on Dynamical Systems-Theory and Applications, 7-10 December, Lodz, Poland, vol. 2, pp. 523-532 (2009)

34. Bensoussan, A., Lions, J.-L., Papanicolaou, G.: Asymptotic Analysis for Periodic Structures. North-Holland, Amsterdam (1978)

35. Kolpakov, A.G., Rakin, S.I.: Calculation of the effective stiffnesses of the corrugated plates by solving the problem on the plate cross-section. J. Appl. Mech. Tech. Phys. 57(4), 757-767 (2016)

36. Babuska, I.: The computational aspects of the homogenization problem. In: Glowinski, R., Lions, J.L., Laboria, I. (eds.) Computing Methods in Applied Sciences and Engineering, 1977, I. Lecture Notes in Mathematics, vol. 704, pp. 309-316. Springer, Berlin (1979)

37. Manevitch, L.I., Andrianov, I.V., Oshmyan, V.G.: Mechanics of Periodically Heterogeneous Structures. Springer, Berlin (2002)

38. Andrianov, I.V., Awrejcewicz, J., Diskovsky, A.A.: Homogenization of quasiperiodic structures. Trans. ASME J. Vib. Acoust. 128(4), 532-534 (2006)

39. Andrianov, I.V., Awrejcewicz, J., Diskovsky, A.A.: Optimal design of ring-stiffened cylindrical shells using homogenization approach. Proc. Inst. Mech. Eng. Part C J. Mech. Eng. Sci. 225(19), 2457-2463 (2011)

40. Andrianov, I.V., Awrejcewicz, J., Diskovsky, A.A.: Sensitivity analysis in design of constructions made of functionally graded materials. Proc. Inst. Mech. Eng. Part C J. Mech. Eng. Sci. 227(1), 19-28 (2013)

41. Andrianov, I.V., Awrejcewicz, J., Diskovsky, A.A.: Functionally graded rod with small concentration of inclusions: homogenization and optimization. Int. J. Non Linear Mech. 91, 189-197 (2017)

42. Banichuk, N.V., Karihaloo, B.L.: On the solution of optimization problems with non-smooth extremals. Int. J. Solids Struct. 13(8), 725-733 (1977)

43. Biderman, V.L.: Mechanics of Thin-Walled Structures. Mashinostroyenie, Moscow (1977). (in Russian) 